Check for updates

Cite this: RSC Adv., 2018, 8, 31008

\title{
Electric heated cotton fabrics with durable conductivity and self-cleaning properties
}

\begin{abstract}
Suhyun Lee and Chung Hee Park (D)*
This study was carried out to improve durability and reduce conductivity degradation of polypyrroledeposited cotton fabrics by introducting a superhydrophobic surface. An in situ polymerization method was used to polymerize the polypyrrole on the cotton fabric, and the surface energy was lowered using $n$-dodecyltrimethoxysilane to create a superhydrophobic surface. In particular, to investigate the durability of the conductivity according to the superhydrophobic surface, the changes of surface resistance were examined after repeated exposure to air, moisture, and friction. The polypyrroledeposited cotton fabric displayed excellent electrical heating features originating from the conductive polymer, although the surface resistance was somewhat increased by the superhydrophobic coating. In addition, nano-roughness was obtained by the pyrrol-deposition on the fabric surface, creating a dualroughness property required for the superhydrophobic surface. Accordingly, the conductive superhydrophobic cotton fabric had a contact angle of more than $150^{\circ}$ and a shedding angle of less than $10^{\circ}$, maintaining superhydrophobicity even during electrical heating. Above all, the superhydrophobic layer contributed to the functional durability of the conductive fabrics by protecting the conductive layer. After atmospheric aging for 20 weeks, undergoing a water spray test for 20 cycles, and a rubbing test with tape, the increment of surface resistance of the superhydrophobic coated cotton fabrics with polypyrrole was increased by up to $30 \%$ compared to the polypyrrole treated specimen without the coating, which showed a decrease of conductivity of over $74 \%$. It is confirmed that the selfcleaning properties can easily remove dirt on the cotton fabric surface by roll-off of water droplets, thereby preventing the degradation of conductivity due to moisture and contamination.
\end{abstract}

Received 28th June 2018

Accepted 27th August 2018

DOI: $10.1039 / \mathrm{c} 8 \mathrm{ra05530k}$

rsc.li/rsc-advances oxygen and moisture, leading to conductivity losses., ${ }^{72}$ Due to its excellent atmospheric stability and high conductivity, polypyrrole is considered a promising candidate for use in conductive polymers. However, ensuring stable conductivity in polypyrrole-deposited fabrics remains a challenge. ${ }^{13}$ Securing the durability of conductivity in the environments of daily use where it may cause contamination and the reduction in the number of launderings is also essential to increase the lifespan of polypyrrole fibre composites.

Superhydrophobicity is defined as a surface that exhibits a static contact angle of more than $150^{\circ}$ and a shedding angle of less than $10^{\circ}$ for water droplets..$^{14}$ Due to its water droplet rolloff characteristics and self-cleaning effects of superhydrophobic surfaces, the removal of dirt is easily achieved. Thus, issues associated with functional degradation caused by dirt or moisture in conductive materials are expected to be solved by the introduction of superhydrophobic surfaces. ${ }^{15-18}$ In addition, it can solve the risk of a short circuit which not only damages the integrated devices, but also endangers the security of wearers due to the wet conductive fabric. ${ }^{19}$

To combine superhydrophobicity and electrical conductive property into the fabrics using polypyrrole, there are two ways; one is to embed doping agents with hydrophobic chemical 
groups into polypyrrole during polymerization, the other is to hydrophobic coating onto the polypyrrole deposited fabrics as a multilayer structure. In the case of the former, there is a problem that polypyrrole is not deposited on the fabric due to the presence of a hydrophobic group, such as fluorinated molecules. In the latter case, the conductivity may decrease due to the hydrophobic layer. ${ }^{\mathbf{2 0 , 2 1}}$ Also, fluorine compounds used for superhydrophobicity are gradually being restricted due to environmental and human safety issues. Therefore, it is necessary to study how to achieve superhydrophobicity by using non-fluorine compounds. ${ }^{22}$

Superhydrophobic surfaces can be implemented physically and chemically through the combination of the micro-nano dual-structure roughness and low surface energies. ${ }^{23-25}$ If an optimal dual-structure roughness is formed, a superhydrohpbic surface can easily be achieved even if the surface energy is not extremely low. The geometric roughness of the surface of conductive polymers can be easily modified because of their unique characteristics. Depending on the polymerization method used (i.e. chemical oxidation polymerization, $\mathbf{4}^{\mathbf{4}, \mathbf{8}, \mathbf{2 6}, 27}$ deposition by electrochemical polymerization, ${ }^{\mathbf{2 8}}$ deposition by vapour polymerization ${ }^{29}$ ) the structure of conductive polymers can be modified on the micro- and nano-levels. The formed structures can be modified in various ways, forming needles, fibres, rods, ribbons, sheet, spheres, hollow spheres, flower-like structures, cauliflower-like structure, and urchin-like structures. ${ }^{14}$ The in situ polymerization method, which is a type of chemical oxidation polymerization, is expected to afford the easy creation of dual roughness because it allows for the polymerization of powder-shaped polypyrrole and instant deposition on the fabric surface.

This study aimed to develop a cotton fabric with selfcleaning property and durable conductivity and electric heating properties by forming a superhydrophobic surface on polypyrrole fibre composites. Polypyrrole was in situ polymerized on the surface of cotton fabrics to introduce conductivity and develop nano-roughness for superhydrophobic surface. Subsequent hydrophobic coating was performed using $n$-dedecyltrimethoxysilane (DTMS) sol-gel solution, creating a functional conductive and superhydrophobic cotton fabric. The conductivity of the polypyrrole deposited and superhydrophobic coated fabric was evaluated in terms of surface resistance and electric heating performance. Superhydrophobicity was examined by the water contact angle, shedding angle and roll-off phenomenon. In order to confirm whether the superhydrophobic surface is effective in protecting the conductivity of the polypyrrole, the change of surface resistance by coating was measured after repeated exposure to air, moisture and friction.

\section{Experimental section}

\section{Material}

Fabrics consisting of $100 \%$ cotton were purchased from Weiqiao Textile Company Ltd. (China). The cotton fabrics were in 16 's plain woven, and their weight and thickness were $188 \mathrm{~g}$ $\mathrm{m}^{-2}$ and $0.42 \mathrm{~mm}$, respectively. The monomer used for the in situ polymerization of the conductive polymer was pyrrole with a 99\% purity (Sigma-Aldrich, USA). Anthraquinone-2-sulfonic acid sodium salt monohydrate with a 97\% purity (SigmaAldrich, USA) was used as a dopant and iron(III) chloride hexahydrate (Kanto Chemical Co., Inc., Japan) as an oxidant. In the surface hydrophobization process, $n$-dedecyltrimethoxysilane (Tokyo Chemical Industry Co., Ltd. Japan), ethyl alcohol anhydrous, 99.9\% (Deajung Chemical \& Metals Co., Ltd. Korea), and a 5 vol\% acetic acid solution (Daejung Chemical \& Metal Co., Ltd. Korea) were used. The chemical were used as- received without further purification.

\section{Polypyrrole deposition}

The polypyrrole-deposited fibre composite was fabricated with guidance from the study of Guangxi Huang et al. ${ }^{8}$ using the in situ polymerization method which deposited a polymerization pyrrole monomer on the cotton fibre surface.

A total of $0.01 \mathrm{~mol}$ of pyrrole monomer and $0.125 \mathrm{~mol}$ of anthraquinone-2-sulfonic acid sodium salt monohydrate (AQSA-Na) dopant were dissolved in $50 \mathrm{~mL}$ of distilled water. A refined cotton fabric sample with dimensions of $7 \mathrm{~cm} \times 7 \mathrm{~cm}$ was immersed in the mixed solution for $20 \mathrm{~min}$ at room temperature to allow the pyrrole monomer to be sufficiently absorbed by the fabric.

Subsequently, $0.01 \mathrm{~mol}$ of iron(III) chloride hexahydrate $\left(\mathrm{FeCl}_{3} \cdot 6 \mathrm{H}_{2} \mathrm{O}\right)$ was dissolved in $50 \mathrm{~mL}$ of distilled water to create an oxidant solution which was added dropwise to the pyrrole monomer mixed solution where the cotton fabric was immersed to initiate polymerization. Since the polypyrrole displays excellent conductivity at polymerization temperature between 10 and $20{ }^{\circ} \mathrm{C},{ }^{1}$ an ice bath was used to decrease the temperature during the polymerization process. The polymerization reactions of the polypyrrole deposition were performed for $1 \mathrm{~h}$ under stirring at $25 \mathrm{rpm}$. After finishing the reaction, the sample was removed, washed with distilled water, and dried at room temperature.

\section{Hydrophobization}

To create a hydrophobized surface on the polypyrrole-deposited cotton fabric, DTMS, which is an alkoxysilane compound, was used. As per the method described by We et al., ${ }^{30}$ DTMS was dissolved in a 75/25 (v/v) ethanol/water solvent to create a $5 \mathrm{vol} \%$ mixed solution, which was hydrolyzed for $48 \mathrm{~h}$ at $35{ }^{\circ} \mathrm{C}$ to created a sol-gel solution. The polypyrrole-deposited fabric was immersed in the sol-gel solution for $200 \mathrm{~s}$ and then squeezed with a roller at $50 \mathrm{psi}$ ( 4 bar) and $15 \mathrm{rpm}$ to remove excess solution. Subsequently, the fabric was cured for $1 \mathrm{~h}$ in an oven at $100{ }^{\circ} \mathrm{C}$.

The polypyrrole deposition and surface hydrophobization process performed in this research to create a conductive fibre composite are shown in Fig. 1.

\section{Conductivity and electric heating property test}

To observe the exterior of the cotton fabric surface after the polypyrrole in situ polymerization process, field emissionscanning electron microscopy (FE-SEM, SUPRA 55 VP, Carl Zeiss, Germany) was used. 


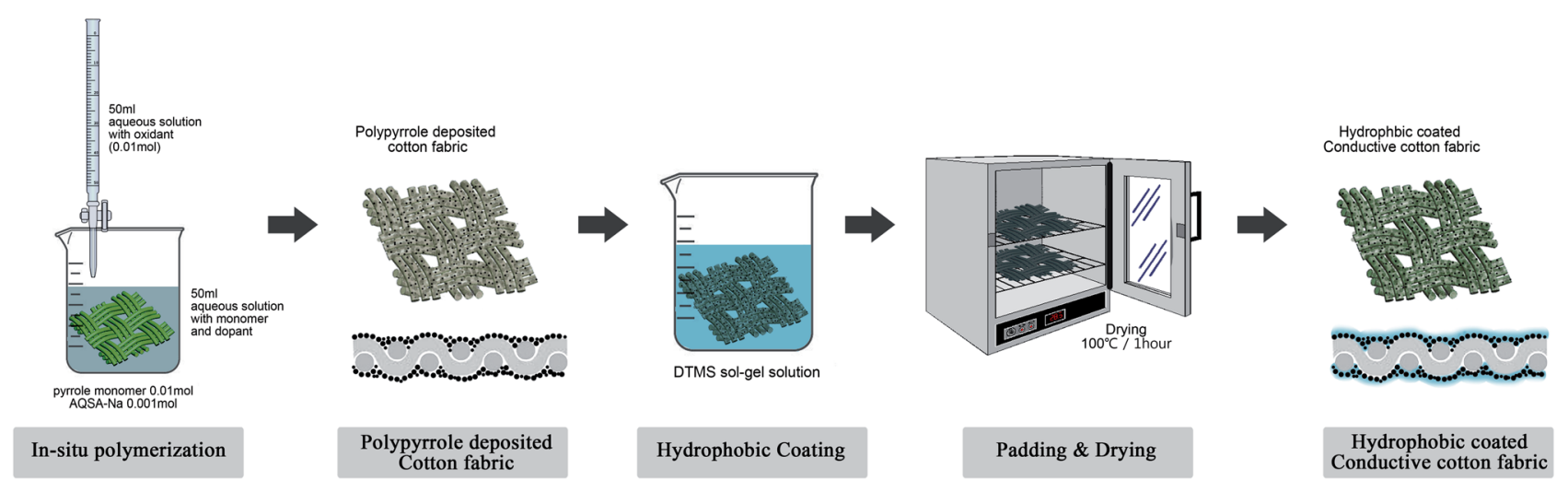

Fig. 1 Scheme of the experimental process.

In accordance with the AATCC 76-1995 method, the electrical surface resistivity of the prepared conductive polymer fibre composite was measured using a DC milliohm meter (GOM804, GW Instek, Taiwan). The final surface resistivity was obtained by performing measurements at 5 different locations on the sample and calculating the average value.

To characterize the electrical heating property according to the resistance heating of the conductive polymer, the surface temperature as a function of time and applied voltage was monitored using a thermal imaging camera (C2, FLIR, USA). The applied voltage was 3, 6, and $9 \mathrm{~V}$ using alkaline batteries (Duracell ${ }^{\circ}$, LR 14 \& 6LF22). The thermal images of the surface temperature were captured in 1 min intervals for a total of $10 \mathrm{~min}$.

\section{Superhydrophobicity and self-cleaning test}

To observe the surface wettability in the untreated cotton fabric, polypyrrole-deposited fabric, and hydrophobized fabric, the static contact angle was measured using a contact angle measuring device (Theta Lite Optical Tensiometer, KSV Instruments, Finland). Each sample was fixed on top of a slide glass and a water droplet with a volume of $3.3 \pm 0.3 \mu \mathrm{L}$ was dropped from $1 \mathrm{~cm}$ above the sample. The contact angle was measured $1 \mathrm{~s}$ after the droplet contacted the surface. The final static contact angle was obtained by repeating this process 10 times at different locations and calculating the average value.

According to the method proposed by Zimmermann et al., ${ }^{31}$ the shedding angle can be defined as the angle at which the drop of water starts rolling down by a distance of $2 \mathrm{~cm}$ after putting the sample on top of an angle adjustable cradle while inclining the measuring plate by $0.5^{\circ}$ at a time. The final value was obtained by performing the same process 5 times at different locations and calculating the average value.

The self-cleaning properties of superhydrophobic surfaces can be measured by simulating the removal of solid particles similar to the way that water droplets that roll off, according to the method proposed by Park et al. ${ }^{\mathbf{1 6}}$ Silicon carbide particles were spread evenly on the sample surface using a mask filter as a sieve and the sample was put on a plat inclined at $10^{\circ}, 7^{\circ}$, and $5^{\circ}$. A water droplet was then added in an identical method as when measuring the shedding angle to confirm the removal of solid particles with the water droplet roll off.

\section{Durability of conductivity test}

To evaluate the oxidation durability of conductive properties, both polypyrrole-deposited and polypyrrole-deposited/ hydrophobic-coated samples were exposed to air at room temperature for 20 weeks. The surface resistance was measured every 2 weeks to monitor conductivity changes over time.

The physical durability of the polypyrrole conductive layer according to the hydrophobicity of the coating was evaluated with a tape test using ASTM D3359. A roll tape cleaner (3 M, Scotch-Brite ${ }^{\mathrm{TM}}$ Tape cleaner) $101 \mathrm{~mm}$ in width was adhered to the prepared sample surface. The tape was adhered to the sample by applying force vertically from left to right, and then removed. This process was repeated 30 times, and the surface resistance, contact angles, and shedding angles were measured every 5 times. The surface resistance and contact angle of the sample after repetitive friction were obtained by performing the experiment in triplicate and calculating the average values.

To examine the effect of the self-cleaning property through hydrophobic coating on conductivity changes caused by repetitive exposure to moisture, the AATCC 22 spray test method, which simulates a self-cleaning environment, was used. The sample was placed on top of a substrate inclined by $45^{\circ}$, and $250 \mathrm{~mL}$ of distilled water was poured on the sample from $15 \mathrm{~cm}$ above it for 25-30 seconds, falling in a spray form. After the spraying the entire volume, the sample was removed, and dried naturally for $30 \mathrm{~min}$. Considering the conductivity improvement effects caused by moisture, the initial surface resistance was selected based on the surface resistance of a sample dried for $30 \mathrm{~min}$ after the spray test to normalize the moisture inside the sample. Conductivity changes after repetitive moisture exposure were observed by repeating the spray test 20 times and measuring the surface resistance every 5 tests.

\section{Results and discussion}

\section{Surface morphologies}

Fig. 2 shows the cotton fabric in which polypyrrole was deposited using chemical oxidation polymerization. Here, it can be 


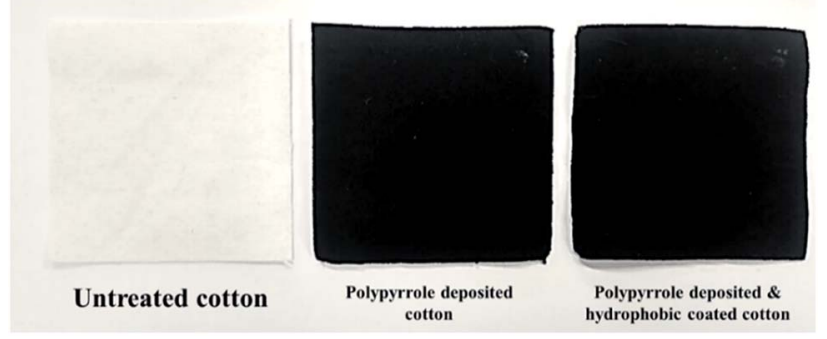

Fig. 2 Photo of polypyrrole deposited cotton samples.

visually verified that the polypyrrole was successfully deposited because after the polymerization process, the color of the cotton fabric became totally black. According to the report by Liu et al., ${ }^{1}$ the polypyrrole and cotton fibre molecules bond through van der Waals attraction and hydrogen bonding.

After depositing polypyrrole on the cotton fabric surface, the surface of the hydrophobized sample was observed using SEM. The untreated cotton fabric exhibited a smooth surface (Fig. 3a and $b$ ), whereas the surface of the polypyrrole-deposited sample is completely covered by small polypyrrole particles $167 \pm$ $27 \mathrm{~nm}$ in size (Fig. 3c and d). The in situ polymerization, which diffuses the conductive polypyrrole polymer inside the fibre, forms nano particles as the pyrrole monomers are polymerized. As a result, the fibre obtains the conductivity by polypyrrole and at the same time develops micro-nano roughness, acquiring the physical roughness necessary for superhydrophobicity. The dual roughness remaining even after the hydrophobic coating is shown in Fig. 3e and f. Therefore, there was no change in the appearance of cotton fabric due to hydrophobic coating.

\section{Surface resistivity and electric heating effects}

The conductivity of polymer develop originate from delocalized charge carriers inside the polymer chain..$^{32}$ Unlike in conventional metals, which are three-dimensional solids, particles that are partially charged by doping display onedimensional electrical conductivity in a direction parallel to the chain of the conductive polymer. ${ }^{33}$ The conductivity of polypyrrole-deposited fibre composites is affected by the polypyrrole deposited on the fabric surface, and by the polypyrrole impregnated deeper inside the fabric. Therefore, the continuity and connectivity of the conductive particles deposited both on the surface and inside the fabric are important factors governing the conductivity of the polypyrrole fibre composites. ${ }^{34}$

To determine the influence of the hydrophobic surface coating and the conductivity imparted by polypyrrole deposition, the surface resistance value was measured after treatment. As shown in Table 1, the surface resistance of cotton could not be measured because it is non-conductive with infinite resistance. However, as shown in Fig. 4, after depositing the polypyrrole on the cotton fabric surface, it acquires conductivity due to the $\pi$-conjugation system of the

Table 1 Surface resistivity of cotton fabrics polypyrrole deposited and hydrophobic coated

\begin{tabular}{lll}
\hline Samples & Surface resistivity $\left(\Omega \square^{-1}\right)$ \\
\hline Untreated cotton & $\infty$ & \\
Polypyrrole deposited cotton & $32 \pm 7$ & $+10.4(32.5 \%)$ \\
Polypyrrole deposited & $42 \pm 7$ &
\end{tabular}
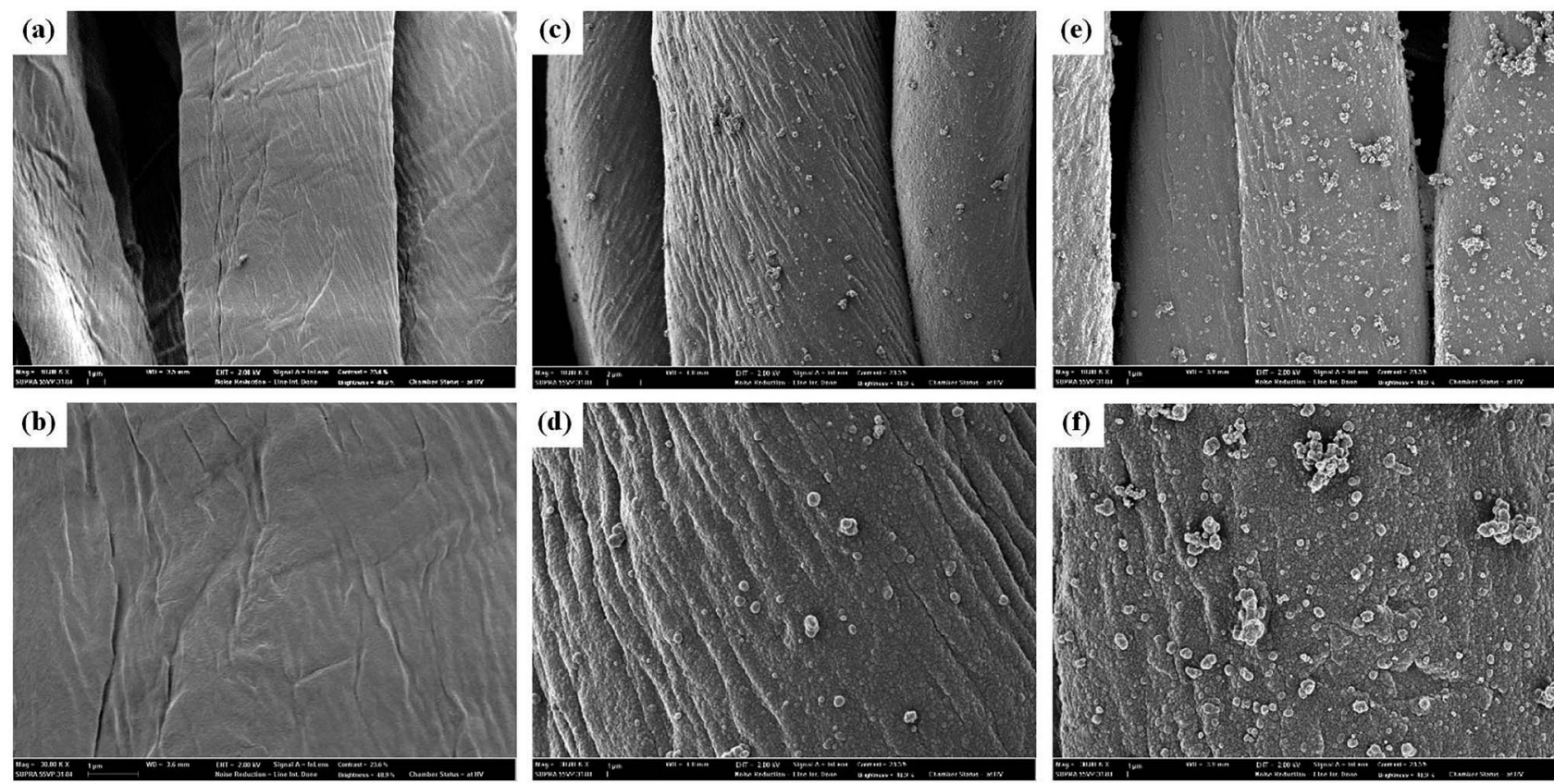

Fig. 3 SEM images of pristine cotton ( $a$ and b), polypyrrole deposited cotton ( $c$ and d) and polypyrrole deposited and hydrophobic coated cotton (e and $\mathrm{f}$ ) 


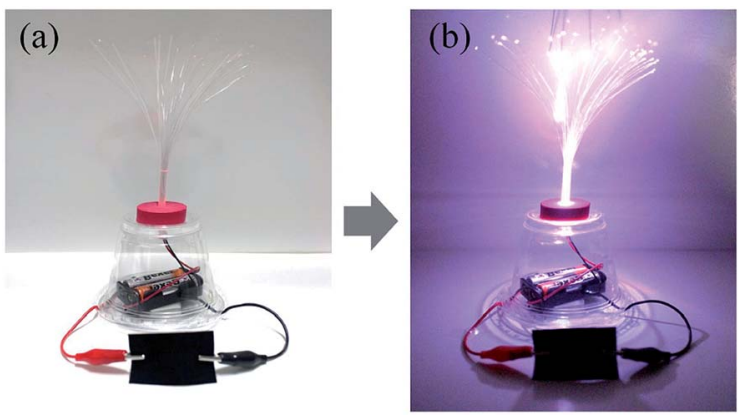

Fig. 4 Photos of LED lamps connected to the polypyrrole deposited and hydrophobic coated cotton fabric.

polypyrrole. The polypyrrole-deposited sample displayed current flow with excellent conductivity due to its very low surface resistance of $32 \pm 7 \Omega \square^{-1}$. Since the cotton fabric was immersed in a pyrrole monomer-dispersed solution, the pyrrole monomers were absorbed sufficiently into the fibre. Then, the monomers reacted with the oxidants, forming polymers and dispersing evenly inside the fibre, forming a continuous conductive layer. ${ }^{1}$ In addition, after the hydrophobic coating was applied, the surface resistance value increased by approximately $32.5 \%$, reaching $42 \pm 7 \Omega \square^{-1}$. The conductive polymer layer was likely covered by the DTMS insulating layer, degrading the continuity between the polypyrrole particles and blocking the path of the electrons. According to Varesano et al. ${ }^{20}$ hydrophobic coating generally resulted in a decrease of conductivity by several orders of magnitude, which means a diminution of electrical performances with respect to neat polypyrrole. In this study, however, the increase in surface resistivity according to the hydrophobic coating was not significant relatively, because the hydrophobic layer was formed thinly on the polypyrrole conductive layer with add-on of less than $1 \%$. Even though, superhydrophobicity was possible with the aid of dual scale roughness minimizing the contact area of liquid drop with fibre surface.

Electric heating by conductive polymers can be described as resistance heating. ${ }^{8-11}$ Generally, when electricity flows through a conductor with resistance, the electric power is converted into heat energy. When electrons pass through the center of conductive polymers, they pass between molecules or atoms due to the applied voltage, in a constant direction causing friction and consuming energy; this consumed energy is converted into heat. ${ }^{35}$ In the heat generating mechanism of the polypyrrole fibre composites, the fibre becomes heated by energy generated from resistance as the electricity flows inside polypyrrole chain, and the generated heat is transmitted by conduction or convection. The thermal efficiency of the fibre varies according to its structure, electricity source, and configuration of the system. ${ }^{11}$

To examine the electric heating characteristics of the prepared polypyrrole-deposited cotton fabrics, surface temperature changes were monitored with a thermo-graphic camera for 10 min after connecting a wire to both ends of the sample and applying a voltage. As shown in Fig. 5, the temperature only

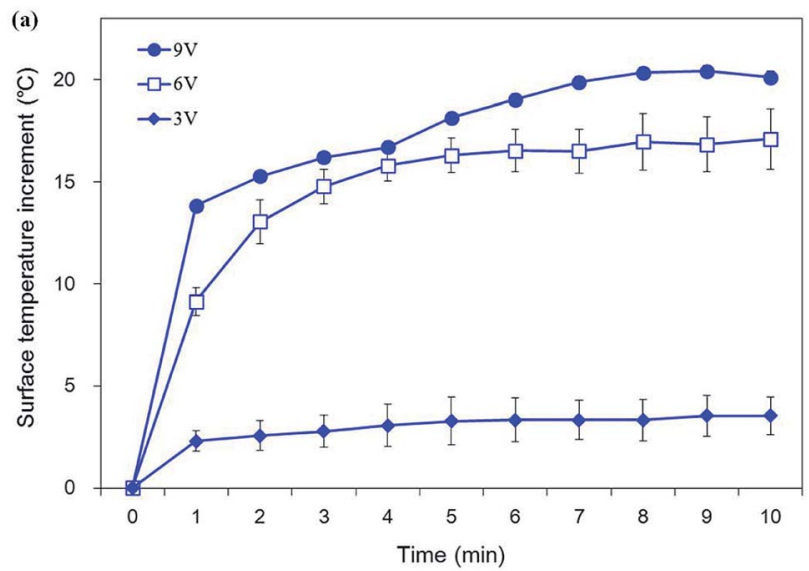

(b)

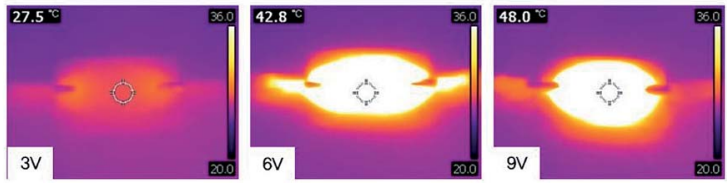

Fig. 5 Surface temperature increment of polypyrrole deposited and hydrophobic coated cotton fabrics (a) and infrared thermal images at $10 \min (b)$.

increased by $3.5^{\circ} \mathrm{C}$, reaching a surface temperature of $27.5^{\circ} \mathrm{C}$ when $3 \mathrm{~V}$ was applied, which is relatively minor. When $6 \mathrm{~V}$ was applied, the temperature increased by $17.1{ }^{\circ} \mathrm{C}$ on average, reaching a surface temperature of $42.8^{\circ} \mathrm{C}$. At $9 \mathrm{~V}$, temperature increased by $20.1{ }^{\circ} \mathrm{C}$, reaching a surface temperature of $48.0^{\circ} \mathrm{C}$ and exhibiting a clear heating effect. Thus, it was verified that the surface temperature increases linearly as a function of applied voltage. In accordance with Joule's law, the amount of current applied to the sample increases with increasing voltage, causing the amount of generated heat to increase proportionally. ${ }^{28}$

Regardless of the voltage applied, all samples displayed a similar electric heating curve as a function of time. In the initial stage (within $3 \mathrm{~min}$ ), the surface temperature increased abruptly in response to the applied voltage. The electric energy delivered to material is reported to exhibit heat losses in the form of convection, radiation, and conduction..$^{36}$ The temperature of the sample increased once the total amount of heat loss was exceeded by resistance heating, and this continued since heating and heat losses achieved a stable equilibrium. According to the conservation of energy, after the temperature rising stage, heating and heat losses caused by convection and radiation are balanced, and a constant temperature is subsequently maintained..$^{28,36-38}$

\section{Superhydrophobicity}

To determine the extent of surface superhydrophobicity developed by the combined hydrophobic coating and nano roughness developed by polypyrrole deposition, the contact and shedding angles were measured. The results of these measurements are shown in Fig. 6.

Cotton is a common hydrophilic fibre containing numerous hydroxyl groups in its molecular chain, which impart good 


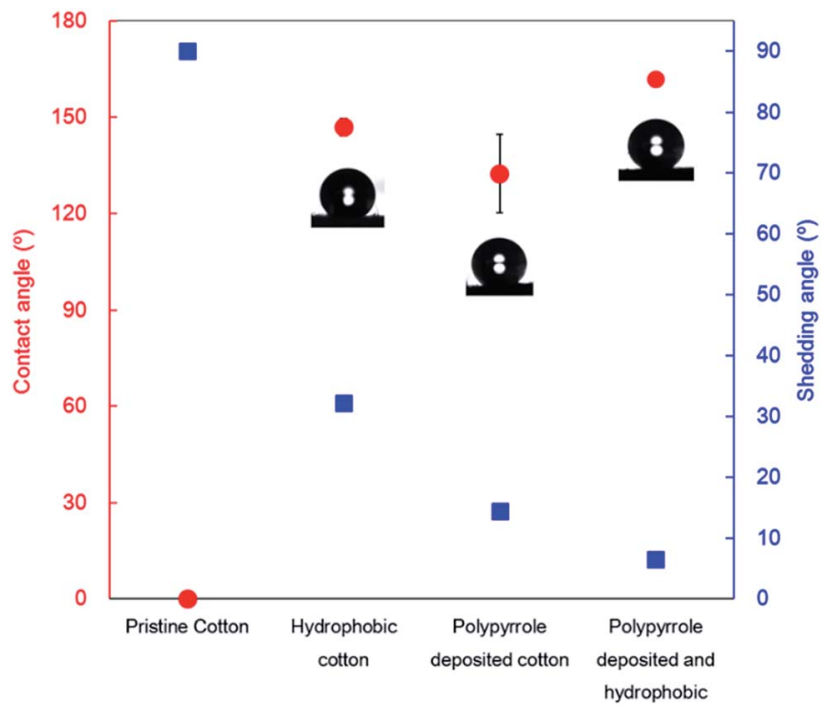

Fig. 6 Water contact angles and shedding angles of the cotton fabrics after polypyrrole deposition and hydrophobic coating.

absorbency to cotton-based materials. Therefore, untreated cotton fabrics absorb water droplets instantaneously, exhibiting a contact angle of $0^{\circ}$, and a shedding angle of more than $90^{\circ}$.

However, when the surface energy of pristine cotton fabrics is reduced through hydrophobic coating, displaying a contact angle of $146.9 \pm 2.8^{\circ}$, and a shedding angle of $32.2 \pm 1.4^{\circ}$. DTMS used for surface hydrophobization decreases surface energy because it contains long alkoxysilane chains that create silica materials upon hydrolysis. ${ }^{39}$ In addition, cotton fabrics have countless $12.5 \mu \mathrm{m}$ staple fibres arranged in a crossed pattern. This creates air pockets between water droplets and fibres, increasing the roughness that improves water repellency. ${ }^{40}$ Therefore, hydrophobicity was achieved only by decreasing the surface energy using DTMS due to the inherent hierarchical roughness of the cotton fabrics composed of yarns and staple fibres. According to Park et al. ${ }^{41}$ in case of fabric, hydrophobicity can be achieved efficiently by treatment with a water repellent coating due to the unique characteristic surface structure of the fabric. They also emphasized that in order to achieve superhydrophobicity on the fabric surface, the physically micro- and nano-dual roughness must be introduced.

After polypyrrole deposition on the cotton fabric, the contact angles and shedding angles were $132 \pm 12^{\circ}$ and $14.4 \pm 0.2^{\circ}$, respectively, showing improved hydrophobicity compared to pristine cotton. Generally, undoped neutral polypyrrole has a rather low surface energy of approximately $32.1 \mathrm{~mJ} \mathrm{~m}^{-2}{ }^{42}$ Therefore, we can see simple polypyrrole coating on the surface of the cotton fabric increased the contact angles and decreased shedding angle by combining the natural micro roughness of cotton fabrics with polypyrrole, developing nano roughness resulting in dual roughness of the prepared material. However, it is also difficult to achieved superhydrophobicity because the surface energy is not sufficiently low. (a)

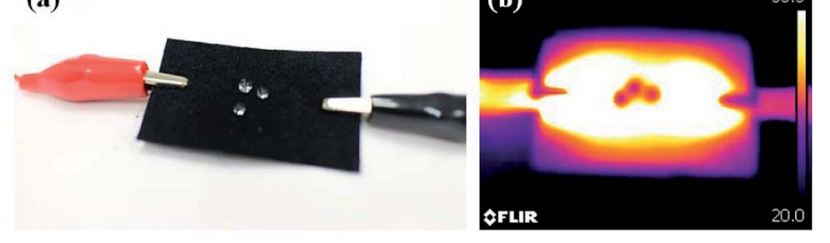

Fig. 7 The photo (a) and infrared thermal image (b) of sample with electro heating effect and superhydrophobicity.

It was confirmed that by decreasing the surface energy of the polypyrrole-deposited cotton fabrics by hydrophobic coating, all physical and chemical conditions required for a superhydrophobic surface were met, as evidenced by the contact angles and shedding angles. The final contact angles were $162 \pm$ $2^{\circ}$ and the shedding angles were $6.5 \pm 0.1^{\circ}$, surpassing that of standard of superhydrophobic surfaces (more than $150^{\circ}$ contact angle and less than $10^{\circ}$ shedding angle). As shown in Fig. 7, the surface maintains its superhydrophobicity even upon heating with current passing through the sample.

\section{Self-cleaning effects}

Superhydrophobic surfaces have self-cleaning properties that facilitate the easy removal of dirt due to the water droplets rolloff. These surfaces can be cleaned by water droplets even at small slope angles due to their superphydrophobicity. ${ }^{43-47}$ This not only prevents the oxidation of polypyrrole by moisture, but also reduces the surface resistivity increase due to contaminants (dirt, soil, etc.) during daily usage. ${ }^{19}$ To achieve this selfcleaning property, both high contact angle and low contact angle hysteresis are required. ${ }^{16-18,41,48-50}$

To evaluate the self-cleaning properties of the fabricated superhydrophobic surface, water droplets were dropped on the sample after sprinkling silicon carbide particles on the surface to observe the removal of particles by the water droplets. Fig. 8 shows images captured every $0.5 \mathrm{~s}$ after filming a video of the self-cleaning phenomenon according to tilting angles.

The self-cleaning test results revealed that the silicon carbide particles were effectively removed from the cotton fabric surface

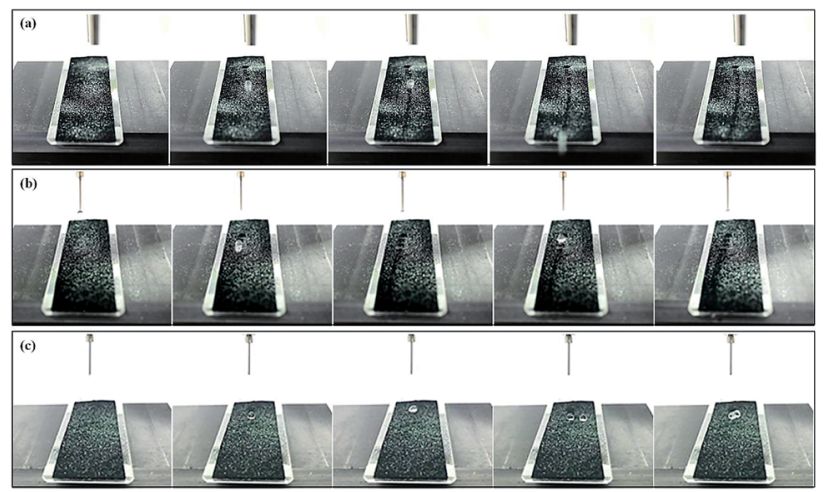

Fig. 8 Images of self-cleaning effect by water at tilting angle (a) $10^{\circ}$, (b) $7^{\circ}$, and (c) $5^{\circ}$. 


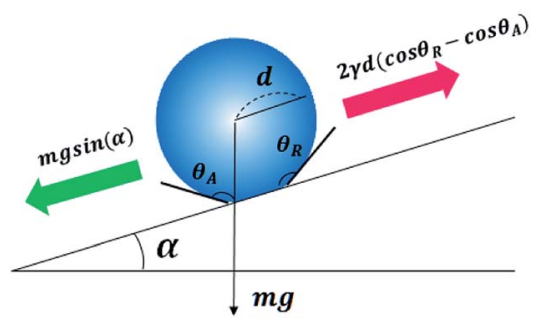

Fig. 9 Schematic view of water droplet on a tilted surface.

by rolling-off water droplets. The roll-off distance of water droplets was more than $2 \mathrm{~cm}$. As shown in Fig. 9, the roll-off water droplet is governed by the gravity applied to droplets and the pinning force between the solid surface and water droplets.

When the water droplets do not roll off the solid surface, the equation describing the gravity and pinning force can be expressed as shown in eqn (1): $:^{4,46}$

$$
m g \sin (\alpha)=2 \gamma d\left(\cos \theta_{\mathrm{R}}-\cos \theta_{\mathrm{A}}\right)
$$

where $m$ is the mass of the water droplet, $g$ is the gravitational acceleration, $\alpha$ is the tilting angle of the surface, $\gamma$ is the surface tension of the water droplets, $d$ is the radius of the droplet, and $\theta_{\mathrm{A}}$ and $\theta_{\mathrm{R}}$ are the advancing and receding contact angles, respectively. According to the equation, if the contact angle hysteresis is low or the surface tilting angle is large, the gravity function becomes larger than the pinning force, causing the water droplets to roll-off. On the other hand, if the contact angle hysteresis is large or the surface tilting angle is low, the pinning force becomes larger than the gravitational force, causing the water droplets to remain still without rolling off.

Therefore, since the slope of the surface tilted by $10^{\circ}$ is higher than the inherent shedding angle of the sample, the gravitational force acts with a greater strength than the adhesive force between the water droplets and sample surface. Even when the slope of the surface tilted by $7^{\circ}$, the superhydrophobic polypyrrole fibre composites exhibited a self-cleaning phenomenon as the water droplets rolled off more than $2 \mathrm{~cm}$ as in the $10^{\circ}$. However, it could not be observed the self-cleaning effect at tiling angle $5^{\circ}$ through the water droplets fell down only to $1.2 \pm 0.3 \mathrm{~cm}$ on the surface, confirming that the shedding angle of the samples is more than $5^{\circ}$.

In addition, the silicon carbide particles used to simulated dirt are hydrophilic and have a greater affinity towards water droplets than superhydrophobic surfaces. ${ }^{16}$ As shown in Fig. 10, the solid particles were absorbed by the water droplets and removed from the surface.

Through this experiment, the self-cleaning properties of polypyrrole-deposited hydrophobized cotton fabrics were confirmed. These properties are expected to contribute to maintaining the functionality of the prepared fabrics by protecting the conductive layer from moisture and contaminants while extending the lifetime of the material by reducing its washing frequency.

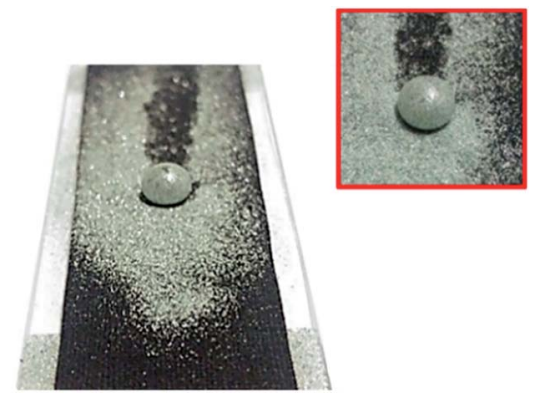

Fig. 10 Photos of the polypyrrole deposited and hydrophobic coated cotton fabric after scattering silicon carbide particles on the surface and cleaning with water droplet.

\section{Durability of conductivity}

Moss \& Burford ${ }^{51}$ identified a number of factors that cause degradation in the stability of conductive polymers. Oxygen can attack the $\alpha$-carbon or $\beta$-carbon of the polypyrrole, cutting the polymer chain, and shortening its conjugation length. ${ }^{52}$ In addition, once oxygen enters the polypyrrole chain, it attacks the $\beta$-position, creating hydroperoxyl radicals (eqn (2)-(4)), causing fracture of the carbon backbone, and loss of free radicals, which degrades the conductivity of the polypyrrole. Furthermore, the decomposition of polypyrrole hydroperoxides generates carbonyl, ester, and hydroxyl groups (eqn (4)). ${ }^{51,53}$ There is also an irreversible loss of the conjugated structure and conductivity in the polymer chain due to reaction with oxygen. In addition, even if the conjugated structure is unaffected, oxygen can react with nitrogen oxides in hetero atoms on the chain, leading to changes in the electronic structure of the polymer. $^{51}$

$$
\begin{gathered}
\mathrm{Py}^{\bullet}+\mathrm{O}_{2} \rightarrow \mathrm{PyOO}^{\bullet} \\
\mathrm{PyOO}^{\bullet}+\mathrm{PyH} \rightarrow \mathrm{PyOOH}+\mathrm{Py}^{\bullet} \\
\mathrm{PyOOH} \rightarrow \mathrm{C}=\mathrm{O}+\mathrm{C}-\mathrm{OH}
\end{gathered}
$$

Therefore, minimizing the contact of conductive polymers with oxygen is expected to mitigate conductivity losses. ${ }^{9,54}$ The hydrophobic coating used in this study introduced superhydrophobicity to the polypyrrole fibre composites and effectively covered the polypyrrole conductive layer, blocking air from penetrating. Thus, it is expected to enhance the durability of the conductivity of the fibre. Additionally, both the polypyrrole-deposited samples and polypyrrole-deposited and subsequently hydrophobic-coated samples were stored at room temperature for 20 weeks to observe changes in their surface resistance as a function of time. Fig. 11 shows the rate of change of the surface resistance value $\left(R_{\mathrm{S}}\right)$ as a function of time with respect to the initial surface resistance value $\left(R_{\mathrm{sp}}\right)$.

The test results revealed that the final surface resistance of the polypyrrole-deposited sample left at room temperature for 20 weeks was $54 \pm 7 \Omega \square^{-1}$, showing an increase of $121 \%$ compared to the initial surface resistance. For the hydrophobiccoated sample, the final surface resistance was found to be $45 \pm$ $7 \Omega \square^{-1}$, only $20 \%$ higher than its initial surface resistance. 


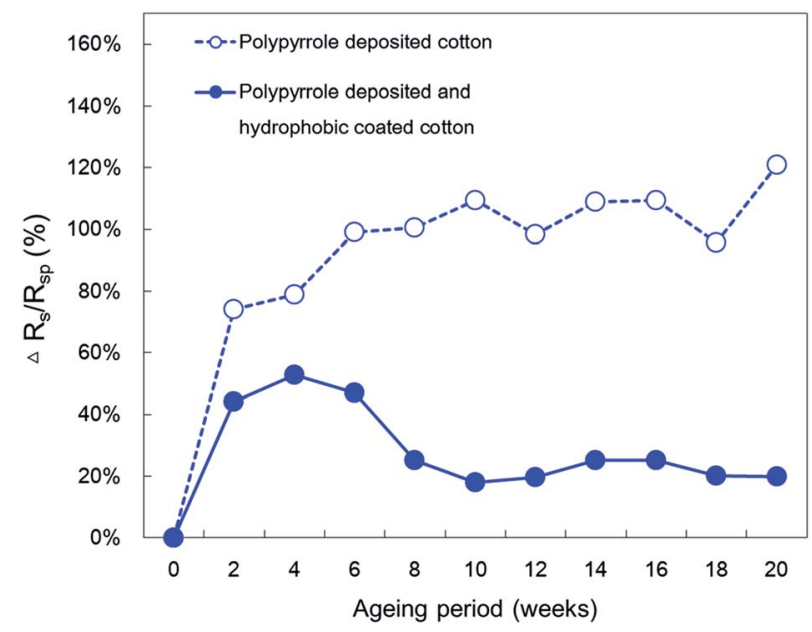

Fig. 11 Effect of atmospheric ageing on the surface resistivity of cotton fabrics treated with polypyrrole deposition and hydrophobic coating.

Thus, the rate of increase in surface resistance of the hydrophobic-coated sample was lower than that of the noncoated sample when exposed to air. The surface resistance of the polypyrrole-deposited sample without hydrophobic-coating increased steadily when left at room temperature for 20 weeks. However, the surface resistance of the hydrophobic-coated sample initially increased and decreased unsteadily until after 8 weeks it reached a constant level, displaying a stable conductivity. Conductivity losses caused by oxidation occur due to the chemical decomposition of the polypyrrole caused by reaction of with oxygen in the air. ${ }^{13,17}$ Therefore when larger amounts of polypyrrole are exposed to air, the durability of the conductivity declines. The polypyrrole-deposited sample without hydrophobic-coating was very reactive because all polypyrrole particles were exposed to air, whereas the hydrophobiccoated sample reacted relatively slowly because the amount of polypyrrole exposed to air at the fibre surface was limited due to the shielding effect of the coating layer. Thus, it was confirmed that the coated hydrophobic layer contributed to enhancing the oxidation durability because it effectively blocks air from contacting the polypyrrole.

Conductivity is well known to be affected by the humidity of the environment. According to Sears, ${ }^{55}$ in humid air there is no significant conduction through the adsorbed water layer itself, but the presence of surface water molecules affects the conductive polymer conduction that occurs through the overlap of adjacent $\pi$-bonds. Especially, he concluded that the hydrophobic nature of conductive polymer makes this more probable, since there would be less water adsorption. This experiment observed the change in conductivity due to atmospheric aging when the polypyrrole deposited fabrics were exposed in a typical environment, such as everyday life. Therefore, it is considered that the temporary decrease of the surface resistance for 4-10 weeks when the humidity was high during the entire experiment period is due to the improvement of the electric current flow by moisture in the air. However, in the case of the uncoated samples, the surface resistance was steadily increased with time regardless of the humidity change of the environment as the polypyrrole degradation by moisture was large enough to override the conductivity improvement effect by the humidity.

The polypyrrole deposited on fibres can fall off from the fabric surface due to external physical forces encountered in daily use. Since this process can also cause conductivity degradation, it is necessary to test for degradation caused by repetitive external forces. In this study, to identify the friction durability of the polypyrrole-deposited cotton fabrics, a tape cleaner test was performed. Previous studies have claimed that commonly used tapes leave glue on the fibre surface, affecting its properties and roughness. ${ }^{56}$ Thus, the test was conducted using a tape cleaner specifically designed for clothing and the results are shown in Fig. 12.

The final surface resistance of the polypyrrole-deposited fabric was $47 \pm 16 \Omega \square^{-1}$, displaying an increase rate of $74 \%$ in surface resistance compared to the initial state. The abrupt surface resistance increase shown in Fig. 12a during the initial 15 tests was a result of polypyrrole particles on the sample surface falling off during the process of attaching and removing the tape cleaner on the sample surface. The surface resistance

(a)

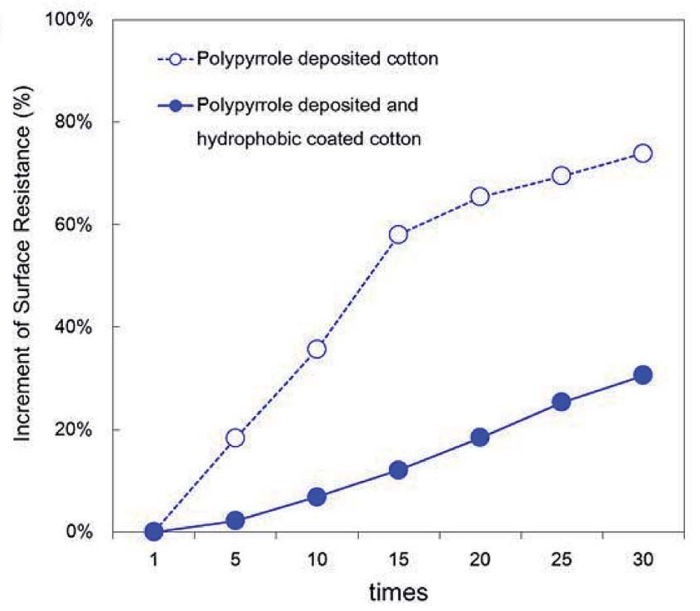

(b)

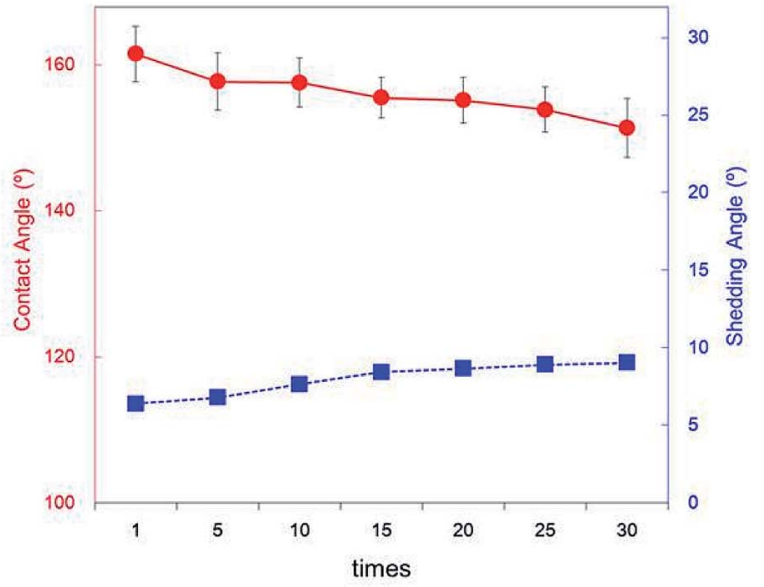

Fig. 12 The rubbing fastness (a), and contact angles and shedding angles (b) of the cotton fabrics treated with polypyrrole deposition and hydrophobic coating after testing with roll tape cleaner. 
curve slope became gentler after the polypyrrole particles with low adhesion strength were removed from the surface. However, on the surface of the hydrophobic-coated sample, the final surface resistance was $43 \pm 5 \Omega \square^{-1}$, achieving an increase of $31 \%$ when compared the initial state. This was caused not only by the hydrophobic coating but also by the padding used during the coating process, which strengthened the adhesion between the cotton and polypyrrole, as 50 psi of pressure was applied to the surface. This process also acted as a binder as the hydrophobic coating layer penetrated the fabric surface and polypyrrole. ${ }^{57}$ In addition, the polypyrrole was prevented from falling off by friction because the surface hydrophobic layer blocked the tape and polypyrrole from coming into direct contact.

As shown in Fig. 12b, the surface wettability after repetitive surface friction changes as the contact angle of the polypyrroledeposited and hydrophobic-coated samples, which has initial contact angles of $161.5^{\circ}$, decreased to $151.4 \pm 4.0^{\circ}$ after frictional forces were applied 30 times. In addition, the shedding angle increased slightly after the friction test, reaching a value of $9.0 \pm 0.3^{\circ}$. Some polypyrrole particles contributing to the nano roughness of the surface fell off due to the friction of the tape, inducing uneven surface roughness changes. Thus, the shedding angle increase was more significant than the contact angle decrease. However, even after repeating the test 30 times, the superhydrophobicity of the surface was retained, with a contact angle of more than $150^{\circ}$ and shedding angle of less than $10^{\circ}$.

Moisture also degrades the conductivity of polypyrroles. ${ }^{13}$ As shown in Fig. 13, water molecules attack the $\alpha$-carbon of the polypyrrole benzene ring to open the ring and rearrange the molecular chains, resulting in irreversible deformation of the polypyrrole polymer chain. ${ }^{\mathbf{1 6}}$ Ensuring the durability of the conductivity against the wet environment that can occur as
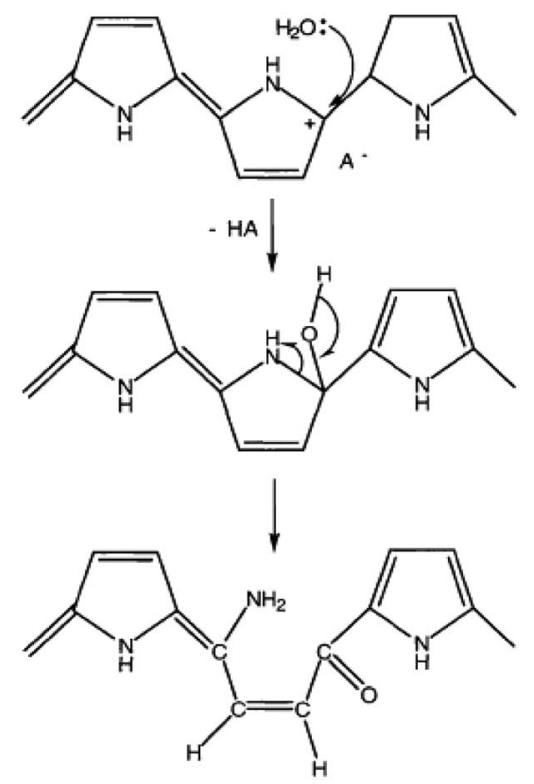

Fig. 13 Water attack at an $\alpha$-carbon on a pyrrole ring leading to chain opening. ${ }^{16}$

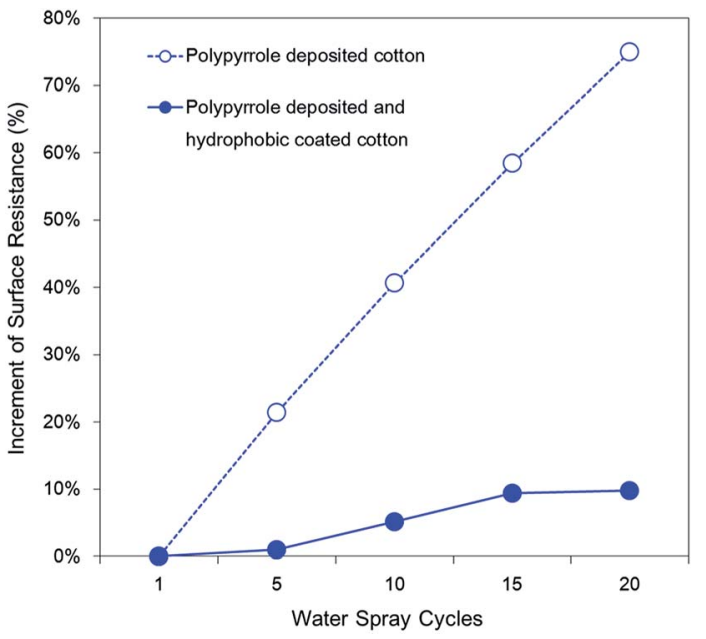

Fig. 14 Effect of moisture on the surface resistivity of the cotton fabrics treated with polypyrrole deposition and hydrophobic coating.

applications for daily products is an important factor in determining the lifetime of the polypyrrole fibre composites. Therefore, it is necessary to examine surface resistance to moistureinduced degradation. Additionally, it is necessary to examine whether the superhydrophobic treatment contributes to maintain the conductivity from repetitive exposure to moisture. Thus, an experiment was conducted using the spray test method and the results are shown in Fig. 14.

The final surface resistance value of the polypyrroledeposited fabric after being exposed to moisture 20 times for a total of $10 \mathrm{~h}$ increased by $75 \%$ compared to the initial value, reaching $38 \pm 5 \Omega \square^{-1}$. For the polypyrrole-deposited sample, an abrupt surface resistance rise was caused by the exposure of the polypyrrole to moisture without protection. Interaction between the polypyrrole chain and water molecules change the electron density of the nitrogen atoms located inside the polypyrrole chain, causing undoping and the possibility of destroying the conjugated structure. ${ }^{16,58}$ For the hydrophobiccoated sample, the final surface resistance increased by only $10 \%$ reaching a value of $39 \pm 5 \Omega \square^{-1}$. The water mostly bounced off the surface due to the excellent water-repellency by superhydrophobicity of the coated sample, preventing moisture from contacting the polypyrrole. Thus, it was confirmed that conductivity losses caused by moisture were delayed by effectively blocking contact of the polypyrrole with moisture using the hydrophobic coating.

Therefore, it is considered that the hydrophobic coating on polypyrrole fibre composites effectively induced superhydrophobicity and improved the conductivity of the polypyrrole by preventing losses caused by exposure of air and moisture.

\section{Conclusions}

In this study, in situ polypyrrole polymerization and superhydrophobic coating was carried out to develop self-cleaning and durable conductive cotton fabrics capable of electric heating. 
The cotton fabrics exhibited electrical conductivity and electric heating performance through polypyrrole deposition. Although the surface resistance was slightly increased by introducing the hydrophobic layer by coating, it was still lower than $50 \Omega \square^{-1}$ which indicated excellent conductivity. In addition, the surface temperature of the coated cotton fabrics with polypyrrole was increased by more than $20^{\circ} \mathrm{C}$ by electric heating performance. Also, nano particles were formed on the fabric surface creating dual roughness through in situ polymerization of polypyrrole, imparting superhydrophobicity to the surface upon application of a simple hydrophobic coating.

The surface hydrophobic coating improved durability of the conductivity by blocking the reaction between polypyrrole and air. The surface resistance of the polypyrrole deposited samples reacted with the air at room temperature for 20 weeks increased by $121 \%$ from the initial value, while the superhydrophobic coated samples increased by only $20 \%$. In addition, as the bonding strength between polypyrrole and the cotton fabric was strengthened through the hydrophobic coating process, the decrease in conductivity due to friction was also improved. In the repetitive rubbing tests, it was shown that the surface resistance of the uncoated samples increased by $74 \%$, while the coated sample increased by $31 \%$. In terms of moisture resistance, the final surface resistivity of the polypyrrole deposited cotton fabric after 20 cycles of water spray test was increased by $75 \%$ from the initial, while the conductivity of the fabric was reduced. However, the conductivity of the superhydrophobic coated samples was maintained, exhibiting only $10 \%$ increase of surface resistance in water spray tests.

Therefore, superhydrophobic surface will make the conductive fabrics easy to manage by self-cleaning property, and contribute to improved durability by protecting the polypyrrole from damage from oxygen, moisture, and external forces.

\section{Conflicts of interest}

There are no conflicts to declare.

\section{Acknowledgements}

This work was supported by a grant (no. NRF2018R1A2B6003526, and NRF-2016M3A7B4910940) of the National Research Foundation (NRF) of Korea funded by the Korean government (Ministry of Science and ICT).

\section{References}

1 Y. Liu, X. Zhao and X. Tuo, J. Text. Inst., 2017, 108, 829-834. 2 C. Sasso, D. Beneventi, E. Zeno, D. Chaussy, M. Petit-Conil and N. Belgacem, BioResources, 2011, 6, 3585-3620.

3 T. Dias, Electronic textiles: smart fabrics and wearable technology, Cambridge, 2016.

4 A. C. Macasaquit and C. A. Binag, Philipp. J. Sci., 2010, 139, 189-196.

5 J. Wu, D. Zhou, C. O. Too and G. G. Wallace, Synth. Met., 2005, 155, 698-701.
6 V. Guarino, S. Zuppolini, A. Borriello and L. Ambrosio, Polymers, 2016, 8, 185, DOI: 10.3390/polym8050185.

7 S. Talha, A. Hamdani, A. Fernando, M. D. Hussain and P. Potluri, J. Ind. Text., 2015, 46, 771-786.

8 G. Huang, L. Liu, R. Wang, J. Zhang, X. Sun and H. Peng, J. Mater. Chem. C, 2016, 4, 7589-7594.

9 A. Kaynak and E. Håkansson, Adv. Polym. Technol., 2005, 24, 194-207.

10 A. C. Sparavigna, L. Florio, J. Avloni and A. Henn, Mater. Sci. Appl., 2010, 1, 253-259.

11 F. Pasila, Y. Tanoto, R. Lim, M. Santoso and N. D. Pah, Proceedings of second international conference on electrical systems, technology and information, Springer, 2015, vol. 35.

12 G. E. Collins and L. J. Buckley, Synth. Met., 1996, 78, 93-101.

13 A. J. Patil and S. C. Deogaonkar, J. Appl. Polym. Sci., 2012, 125, 844-851.

14 T. Darmanin and F. Guittard, Prog. Polym. Sci., 2014, 39, 656682.

15 Y. L. Zhang, H. Xia, E. Kim and H. B. Sun, Soft Matter, 2012, 8, 11217-11231.

16 S. Park, J. Kim and C. H. Park, RSC Adv., 2016, 6, 4588445893.

17 A. Fernández, A. Francone, L. H. Thamdrup, A. Johansson, B. Bilenberg, T. Nielsen, M. Guttmann, C. M. S. Torres and N. Kehagias, J. Micromech. Microeng., 2017, 27, 045020.

18 Q. Xu, W. Zhang, C. Dong, T. S. Sreeprasad and Z. Xia, Interface, 2017, 13, 20160300.

19 X. Li, Y. Li, T. Guan, F. Xu and J. Sun, ACS Appl. Mater. Interfaces, 2018, 10, 12042-12050.

20 A. Varesano, F. Rombaldoni and C. Tonetti, Fibers Polym., 2013, 14, 703-709.

21 D. Mecerreyes, V. Alvaro, I. Cantero, M. Bengoetxea, P. A. Calvo, H. Grande, J. Rodrigues and J. A. Pomposo, Adv. Mater., 2002, 14, 749-752.

22 J. L. Butenhoff and J. V. Rodricks, Toxicological effects of perfluoroalkyl and polyfluoroalkyl substances, 2015, pp. 363418.

23 S. Park, J. Kim and C. H. Park, J. Eng. Fibers Fabr., 2015, 10, 118.

24 W. S. Tung and W. A. Daoud, J. Mater. Chem., 2011, 21, 78587869.

25 I. P. Parkin and R. G. Palgrave, J. Mater. Chem., 2005, 15, 1689-1695.

26 S. C. Deogaonkar and A. J. Patil, Indian J. Fibre Text. Res., 2014, 39, 135-138.

27 X. G. Li, A. Li, M. R. Huang, Y. Liao and Y. G. Lu, J. Phys. Chem. C, 2010, 114, 19244-19255.

28 R. John and G. G. Wallace, J. Electroanal. Chem., 1991, 306, 157-167.

29 H. Wang, H. Zhou, A. Gestos, J. Fang, H. Niu, J. Ding and T. Lin, Soft Matter, 2013, 9, 277-282.

30 L. K. Wu, J. M. Hu, J. Q. Zhang and C. N. Cao, Electrochem. Commun., 2013, 26, 85-88.

31 J. Zimmermann, S. Seeger and F. A. Reifler, Text. Res. J., 2009, 79, 1565-1570.

32 S. Lee, S. K. Lee, D. Jang and B. S. Shim, Elastomers Compos., 2017, 52, 69-80. 
33 A. J. Heeger, S. Kivelson, J. R. Schrieffer and W. P. Su, Rev. Mod. Phys., 1988, 60, 781.

34 N. V. Blinova, J. Stejskal, M. Trchová, J. Prokeš and M. Omastová, Eur. Polym. J., 2007, 43, 2331-2341.

35 Q. A. Acton, Advances in silicon dioxide research and application, Scholarly Editions, 2013 edn, 2013.

36 A. Hebeish, S. Farag, S. Sharaf and Th. I. Shaheen, Carbohydr. Polym., 2016, 151, 96-102.

37 R. K. Singh, A. Kumar, K. Agarwal, D. Dwivedi, K. N. Sood and R. Singh, Open J. Polym. Chem., 2012, 2, 105-112.

38 K. M. Cheung, D. Bloor and G. C. Stevens, J. Mater. Sci., 1990, 25, 3814-3837.

39 L. K. Wu, J. M. Hu, J. Q. Zhang and C. N. Cao, Electrochem. Commun., 2013, 26, 85-88.

40 S. K. Mohammad and E. Y. Mohammad, Cellulose, 2013, 20, 963-972.

41 S. Park, J. Kim and C. H. Park, Text. Res. J., 2017, 82, 193-207.

42 M. J. Higgins and G. G. Wallace, Polym. Rev., 2013, 53, 506526.

43 S. Sutha, S. C. Vanithakumari, R. P. George, U. K. Mudali and B. Raj, Appl. Surf. Sci., 2015, 347, 839-848.

44 J. Toster and D. Lewix, Aust. J. Chem., 2015, 68, 1228-1232.
45 S. T. Larsen, N. K. Andersen, E. Søgaard and R. Taboryski, Langmuir, 2014, 30, 5041-5045.

46 S. A. Jeong and T. J. Kang, Text. Res. J., 2017, 87, 552-560.

47 S. Lee and C. H. Park, Text. Res. J., 2018, 88, 777-789.

48 M. H. Shim, J. Kim and C. H. Park, Text. Res. J., 2014, 84, 1268-1278.

49 Y. Rahmawan, L. Xu and S. Yang, J. Mater. Chem. A, 2013, 1, 2955-2969.

50 J. Zimmermann, S. Seeger and F. A. Reifler, Text. Res. J., 2009, 79, 1565-1570.

51 B. K. Moss and R. P. Burford, Polym. Int., 1991, 26, 225-231. 52 J. Tabačiarová, M. Mičušík, P. Fedorko and M. Omastová, Polym. Degrad. Stab., 2015, 120, 392-401.

53 W. Trevor and G. G. Lewix, Analyst, 1999, 124, 213-219.

54 S. Maity, A. Chatterjee, B. Singh and A. P. Singh, J. Text. Inst., 2014, 105, 887-893.

55 W. M. Sears, Sens. Actuators, B, 2008, 130, 661-667.

56 J. H. Oh, T. J. Ko, M. W. Moon and C. H. Park, RSC Adv., 2017, 7, 25597-25604.

57 G. Liang, L. Zhu, J. Wu, D. Fang, Z. Bai and W. Xu, Electrochim. Acta, 2013, 103, 9-47.

58 J. M. Hu, L. Liu, J. Q. Zhang and C. N. Cao, Prog. Org. Coat., 2007, 58, 265-271. 